\title{
Comparison between thoracoscopic and open approaches in thymoma resection
}

\author{
Guo-Wen Wang, Tao Tao, Chuan-Kui Li, Qi-Cai Li, Gui-Xin Duan, Hai-Wei Sang, Hai-Jun Dong, \\ Zu-Yi Wang
}

Department of Thoracic Surgery, the First Affiliated Hospital of Bengbu Medical College, Bengbu 233000, China

Contributions: (I) Conception and design: GW Wang, ZY Wang; (II) Administrative support: GW Wang, ZY Wang; (III) Provision of study materials or patients: GW Wang, T Tao, GX Duan; (IV) Collection and assembly of data: CK Li, QC Li, HW Sang, HJ Dong; (V) Data analysis and interpretation: GW Wang, HJ Dong, ZY Wang; (VI) Manuscript writing: All authors; (VII) Final approval of manuscript: All authors.

Correspondence to: Zuyi Wang. Department of Thoracic Surgery, the First Affiliated Hospital of Bengbu Medical College, No. 287, Changhuai Road, Longzihu District, Bengbu 233000, China. Email: wgwpc@hotmail.com.

Background: To investigate the feasibility and indications of video-assisted thoracic surgery (VATS) in thymoma resection.

Methods: The clinical data of 103 patients undergoing thymoma resection via different approaches [including conventional lateral thoracotomy approach (LTA) in 41 cases, median sternotomy approach (MSA) in 40 cases, and right-sided VATS in 22 cases] were analyzed. Among them, 59, 13, 25, and 6 patients were in Masaoka stage I, II, III, and IV, respectively. Myasthenia gravis (MG) was also found in 54 cases. The patients were followed up for postoperative survival and the improvement in MG. The prognostic indicators of patients undergoing thymoma resection via different surgical approaches (i.e., LTA, MSA, and VATS) were statistically analyzed.

Results: Eight of 103 patients died. Six patients underwent unilateral sacral nerve resection, among whom 4 patients developed respiratory dysfunction, and 3 died. Two patients died of MG after surgery, 1 patient died of tumor recurrence and metastasis, 1 patient died of heart disease, and the cause of death was unknown in the remaining patient. The drainage time was shorter in VATS group than in open groups, along with smaller tumor size. The VATS group also had shorter hospital stay in the whole series and the subgroup without accompanying MG. The improvement in MG showed no significant difference among the three surgical groups. Both 5- and 10-year survival rates were $91 \%$ in the entire cohort.

Conclusions: VATS is like a conventional surgeries for improving MG in thymoma patients with accompanying MG. VATS resection can still be considered for thymoma that only invades the mediastinal pleura. For thymomas that have intact capsules and have not invaded mediastinal pleura, MSA surgery shall be performed to ensure patient safety if the anteroposterior diameters of the tumors are large and the masses have produced severe compression of the innominate vein, even if the tumors are still in the Masaoka stage II. For thymomas with large left-to-right diameters and with most parts of the tumors located in the left thoracic cavity, a left-sided approach (either VATS or an open approach) may be used in the absence of MG; if MG accompanies the condition, an MT approach or a bilateral VATS may be considered. In patients with unilateral pericardial phrenic nerve and/or local pericardial involvement, right-sided VATS thymectomy may be considered for thymomas located at the right side and bilateral VATS surgery can be performed for tumors located at the left side. In summary, VATS is feasible for the treatment of thymoma complicated by MG. VATS can be performed in patients with Masaoka stage I, II and (a certain portion of) III thymoma; for some patients with Masaoka stage II thymoma, especially those with compression of the innominate vein, the use of VATS should be cautious.

Keywords: Thymoma; myasthenia gravis (MG); video-assisted thoracic surgery (VATS)

Submitted Sep 18, 2019. Accepted for publication Sep 30, 2019.

doi: $10.21037 /$ jtd.2019.09.85

View this article at: http://dx.doi.org/10.21037/jtd.2019.09.85

(c) Journal of Thoracic Disease. All rights reserved. 


\section{Introduction}

Thymoma is the most common mediastinal tumor and accounts for nearly $20 \%$ to $40 \%$ of all types of mediastinal tumors in adults. According to the data released by the US National Cancer Institute, the overall population incidence rate is about $1.5 / 1$ million (1). The surgical resection rate is $100 \%$ for Masaoka stage I and II thymomas. For stage III tumors that invade the mediastinal pleura and adjacent organs, most of them can be cured by extended thymectomy, with a survival rate similar to those of patients with stage I and II thymomas. Therefore, surgery is the treatment of choice for all resectable thymomas (including those can be treated with extended resection). For most stage I and II thymomas and some of stage III thymomas that have invaded the mediastinal pleura and for patients with accompanying myasthenia gravis (MG), is routine thoracotomy along with extended anterior mediastinal fat dissection still required? With the wide application of video-assisted thoracoscopic surgery (VATS), it is also questionable whether it differs from the traditional surgery in terms of thymectomy indicators and prognosis. In this study, we summarized our recent experience in VATS thymectomy and traditional open approaches, with an attempt to find a more solution.

\section{Methods}

\section{General data}

The clinical data of 103 patients undergoing thymoma resection via different approaches in our center from January 2007 to June 2015 were retrospectively analyzed. All the thymomas were pathologically confirmed after surgery. The patients included 61 males and 42 females aged $18-77$ years $[(52.10 \pm 12.005)$ years]. The co-morbidities included MG in 54 cases, anemia in 5 cases, and hypoproteinemia in 1 case. According to the Masaoka criteria, there were 59 patients in stage I, 13 in stage II, 25 in stage III, and 6 in stage IV. Eight patients died during the observation period, including 3 perioperative deaths and 4 deaths noted three months after surgery.

\section{Preoperative preparations}

All patients underwent contrast-enhanced chest CT before surgery to locate the tumor and identify its relationships with surrounding tissues.

The diagnosis of MG was based on the following three criteria: (I) disorder of the postsynaptic membrane at the neuromuscular junction, as confirmed by repetitive nerve stimulation; (II) with clinical symptoms of muscle weakness, including drooping eyelids, diplopia, decreased forces in limb muscles, and difficulty in swallowing and breathing; and (III) responsive to acetylcholinesterase inhibitors (e.g., pyridostigmine bromide). The typing of all myasthenia cases was based on the Myasthenia Gravis Foundation of America (MGFA) criteria.

All MG patients, naive to pyridostigmine bromide or not, were orally administered with prednisolone $60-80 \mathrm{mg} / \mathrm{d}$ one week before surgery. Oral prednisolone at the same dose continued on the operative day surgery and after surgery. The symptoms disappeared after three months, and the dose of prednisolone was gradually tapered down by $5 \mathrm{mg}$ a month until it was completely withdrawn. For patients whose symptoms could not be controlled or even aggravated, azathioprine or cyclophosphamide $(50 \mathrm{mg} / \mathrm{d})$ was added and prednisone $(5 \mathrm{mg} / \mathrm{m})$ was tapered.

\section{Surgical methods}

Based on the results of medical imaging, an agreement was reached on the surgical method for each patient among the department members. For tumors that might have invaded blood vessels and surrounding tissues, median sternotomy approach (MSA) was applied; for large tumors that had compressed the adjacent tissues or inpatient whose specimens were difficult to remove via endoscope, MSA or lateral thoracotomy approach (LTA) was used. Other patients were treated with a randomly selected approach. Finally, there were 40 patients in the MSA group, 41 in the LTA group, and 22 in the VATS group. Other procedures included partial resection of bilateral upper lungs $(n=1)$, partial resection of left upper lung $(n=1)$, resection of left innominate vein $(n=3)$, resection of right innominate vein $(\mathrm{n}=1)$, resection and reconstruction of left innominate vein $(n=1)$, and unilateral sacral nerve resection $(n=6)$. All patients in the MSA group received anterior mediastinal fat dissection, during which adipose tissue was removed. The bilateral sacral nerves were reached on both sides, and the extent as follows: left and right sides: to the pericardial sacral nerve level; lower border: to the adipose tissue at the palpebral angle; and the upper border: to the inferior thyroid plexus. All patients in the LTA group and VATS group underwent unilateral fat dissection and partial resection of adipose tissue, with the extent as follows: the upper border: to the inferior thyroid plexus; the lower 
border: to the palpebral angle; and the lateral side: to the sacral nerve.

\section{Postoperative adjuvant therapy}

For patients with type B2, B3, or C thymoma and in Masaoka stage III or IV, radio chemotherapy was routinely performed after surgery.

\section{Follow-up}

All patients were followed up by telephone and outpatient visits for 14-115 months. The deadline for the follow-up was August 2016. The examinations during the follow-up included: (I) did MG occur in patients without MG before operation; (II) in MG patients, the outcome was evaluated according to drug administration before and after surgery and the MGFA clinical classification as complete remission (CR), partial remission (PR), stable disease (S), and aggravation (A); and (III) in patients with chest discomfort, chest CT scan was performed to determine whether the tumor had recurred.

\section{Statistical analysis}

The statistical analysis was performed using the SPSS 17.0 software package. The clinicopathological factors and survival status were compared by using the chi-square test, $t$-test, and analysis of variance (ANOVA) as well as life-table method. A value of $\mathrm{P}<0.05$ was considered statistically significant.

\section{Results}

Eight of 103 patients died. Six patients underwent unilateral sacral nerve resection, among whom 4 patients developed respiratory dysfunction, and 3 died. Two patients died of MG after surgery, 1 patient died of tumor recurrence and metastasis, 1 patient died of heart disease, and the cause of death was unknown in the remaining patient.

CT scans during the follow-up revealed tumor recurrence in 5 patients, among whom 2 patients underwent reoperation and 3 continued to receive radiotherapy. Since the other patients failed to receive regular CT scans during the follow-up, it was not possible to identify asymptomatic patients with local recurrence.

The relationships between surgical approaches and clinicopathological factors in the whole series and patients with or without MG are shown in Tables 1-3.

As shown in these tables, the drainage time was shorter in the VATS group than in open groups, along with smaller tumor size. The VATS group also had shorter hospital stay in the whole series and the subgroup without accompanying MG. In subgroup with accompanying MG, control of MG was one of the main therapeutic targets, and thus the hospital stays showed no significant difference.

In the thymoma subgroup with accompanying MG $(\mathrm{n}=54)$, the improvement in $\mathrm{MG}$ and its relationships with all clinicopathological factors are summarized in Table 4.

As shown in Table 4, the improvement in MG showed no significant differences among three surgical groups.

The survival rate showed no significant difference during the follow-up period. Because the patients did not receive a regular chest CT scan, it was not possible to determine the local recurrence in all patients.

\section{Discussion}

Complete thymectomy by open sternotomy is traditionally the standard treatment for both benign and malignant thymomas (2-4). In particular, for patients with thymic masses without complete capsules accompanied by myasthenia, median sternotomy may enable a more complete and thorough thymectomy (5). Since median sternotomy is associated with relatively large surgical trauma, lateral thoracotomy for thymoma resection has also been applied, along with the application of a variety of minimally invasive surgical procedures such as transcervical incision, VATS, and robotic surgery (11). However, concerns on the thoroughness of surgery have limited the use of minimally invasive thymectomy. Nevertheless, the minimally invasive techniques have increasingly been adopted as they can reduce the intraoperative complications and deaths, decrease trauma, alleviate postoperative pain, and shorten the length of hospital stay.

As a minimally invasive surgery, VATS is relatively easy to be adopted. However, its role in thymoma resection remains controversial. The use of VATS thymectomy in patients without MG was described over two decades ago (12). According to literature, VATS is safe and feasible for the treatment of early and small thymomas (2,13-15). Meanwhile, some studies have shown that VATS can achieve good oncological prognosis in the treatment of thymomas (16-18). However, these studies often have certain limitations such as early disease stages and lack of comparison with conventional thoracotomy. Odaka et al. 
Table 1 Relationship between different surgical approaches and clinical pathological factors in 103 patients with thymoma

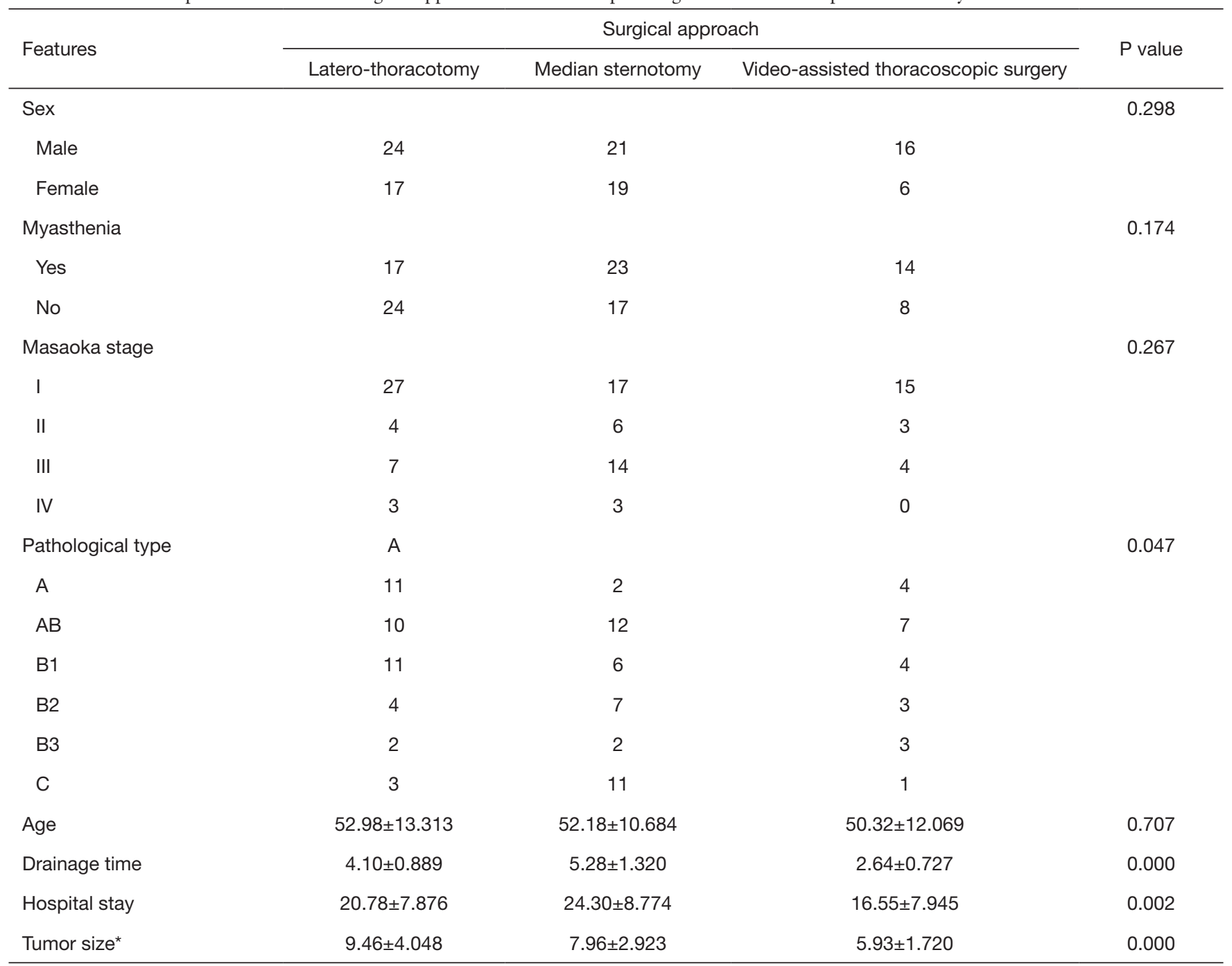

${ }^{*}$, the longest diameter of the tumor.

suggests that while VATS is technically safe for thymoma resection, there is still a lack of oncological data on longterm effectiveness (19). The improvement of the co-existing MG is an important indicator for prognosis. As shown in our current series, there was no significant difference in all clinicopathological factors except for the tumor size between VATS group and open surgery groups. In clinical settings, only a small proportion of patients have thymomas that severely affect the surrounding tissues and organs. Except for these patients, patients in the VATS group in our current study were selected randomly, and the safety of surgery in the VATS group could be guaranteed.

Furthermore, the VATS group had smaller extent of surgical resection than the MS group and showed certain superiority over the LS group. While the improvement of MG was similar between VATS group and conventional thoracotomy groups, the drainage duration was significantly shortened, and the wound pain was significantly alleviated in the VATS group. It has been reported that the thoroughness of the resection of the thymus and its surrounding adipose tissue is related to the improvement of postoperative MG symptoms (20), and some authors have even proposed that an additional neck incision should be created to dissect the adipose tissue at the root of the neck. In our current study, however, the improvement in MG showed no significant difference among three groups undergoing complete 
Table 2 Relationship between different surgical approaches and clinical pathological factors in 54 patients with thymoma complicated with myasthenia gravis

\begin{tabular}{|c|c|c|c|c|}
\hline Features & \multicolumn{3}{|c|}{ Surgical approach } & $P$ value \\
\hline Sex & & & & 0.621 \\
\hline Male & 9 & 11 & 9 & \\
\hline Female & 8 & 12 & 5 & \\
\hline I & 4 & 8 & 1 & \\
\hline Ila & 9 & 5 & 7 & \\
\hline $\mathrm{Ilb}$ & 4 & 6 & 6 & \\
\hline III & 0 & 4 & 0 & \\
\hline II & 1 & 2 & 2 & \\
\hline III & 4 & 7 & 2 & \\
\hline IV & 1 & 0 & 0 & \\
\hline Pathological type & & & & 0.511 \\
\hline$A$ & 4 & 2 & 2 & \\
\hline$A B$ & 5 & 8 & 5 & \\
\hline B1 & 4 & 3 & 4 & \\
\hline B2 & 2 & 5 & 1 & \\
\hline $\mathrm{CR}$ & 6 & 8 & 4 & \\
\hline$A$ & 5 & 6 & 3 & \\
\hline Age & $52.00 \pm 11.486$ & $50.78 \pm 9.643$ & $45.5 \pm 9.788$ & 0.184 \\
\hline Diseased time & $7.69 \pm 11.469$ & $6.04 \pm 10.805$ & $1.47 \pm 1.647$ & 0.192 \\
\hline Drainage time & $4.12 \pm 0.697$ & $5.43 \pm 1.273$ & $2.93 \pm 0.730$ & 0.000 \\
\hline Hospital stay & $19.82 \pm 5.615$ & $24.00 \pm 7.58$ & $20.57 \pm 7.208$ & 0.136 \\
\hline Tumor size ${ }^{\star}$ & $8.79 \pm 4.228$ & $7.17 \pm 3.059$ & $5.68 \pm 1.846$ & 0.035 \\
\hline
\end{tabular}

*, the longest diameter of the tumor. MG, myasthenia gravis; S, stability; PR, partial remission; CR, complete remission; A, aggravation. 
Table 3 Relationship between different surgical approaches and clinicopathological factors in 49 patients with myasthenia thymus

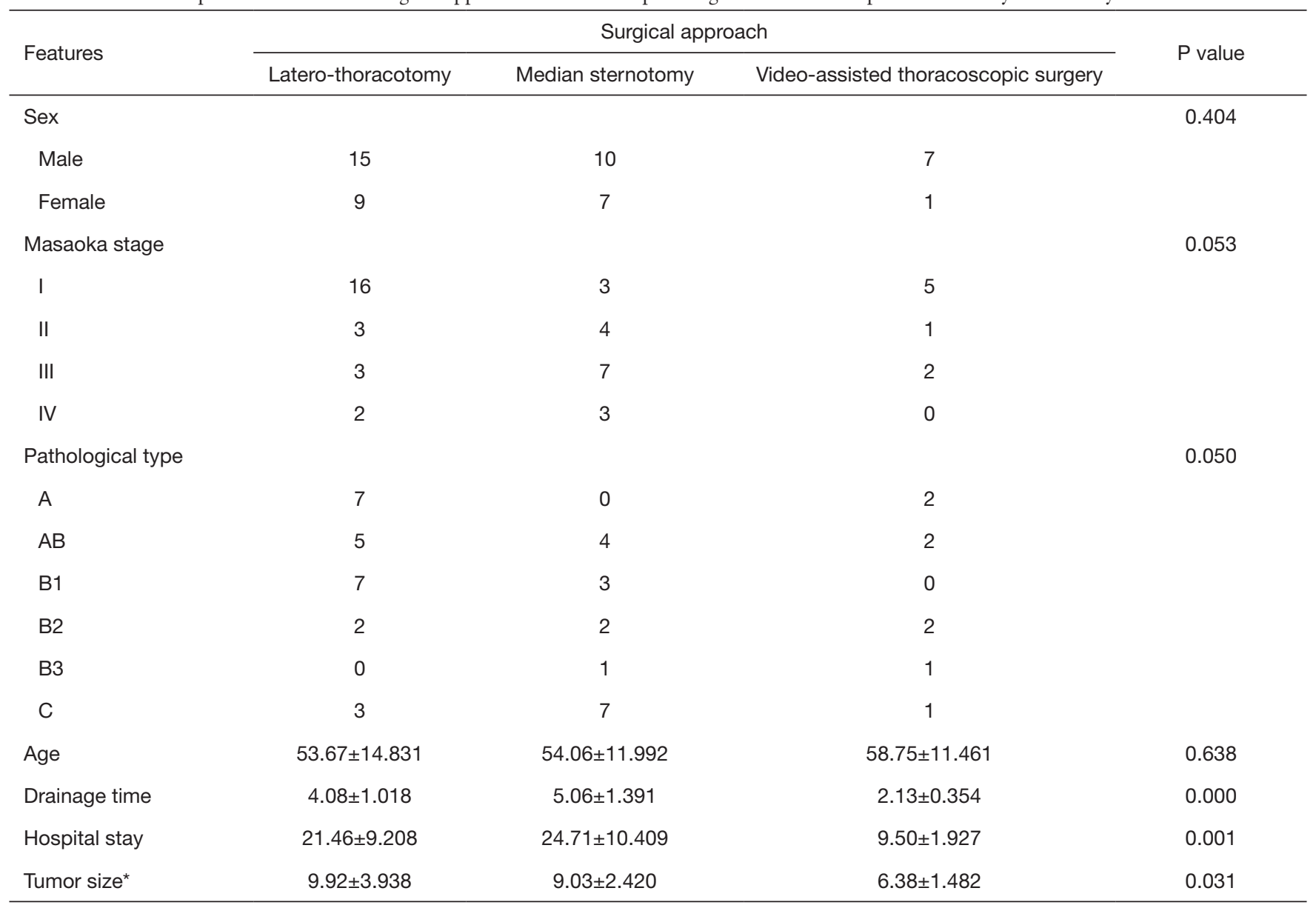

${ }^{*}$, the longest diameter of the tumor.

dissection of anterior mediastinal adipose tissue via different incisions. This might be due to the small sample size and needed to be further validated.

According to the International Thymic Malignancy Interest Group (ITMIG) guidelines, even early thymoma patients without MG should undergo complete resection (CT) (i.e., resection of thymoma and all the glandular lobes). However, multicenter studies in Korea and Japan $(22,23)$ compared the outcomes of patients with stage I or II thymoma treated with limited resection (LT) (i.e., resection of thymoma and parts of the thymus or adipose tissue around the tumor) were not significantly different from those of stage I/II patients treated with CT. Other singlecenter studies also did not find any significant difference between LT and CT in terms of survival and recurrence (24-27). In our current study, all thymoma patients in our center during the study period were included, with an attempt to further explore the prognostic impacts of LT and
CT in patients at different stages. The resection extent for LT group and VATS group can be regarded as LT, whereas that for MT group as CT. A total of 31 patients had stage III or IV thymoma, among whom 13 also had MG (Table 5). Among the patients with imaging data, 5 patients (1 in LT group, 1 in VATS group, and 3 in MT group) experienced recurrence. Eight patients died during the observation period, including 3 perioperative deaths ( 2 in MT group and 1 in LT group). The remaining two deaths included patient with median chest surgery died of tumor recurrence and metastasis after 13 months. Another case of laparoscopic surgery patients died of heart disease. Among the five dead patients, three had type $\mathrm{C}$ thymoma, 1 had type B2 thymoma, and 1 had type B3 thymoma. According to currently available literature, the prognosis is mainly affected by pathological stage and histological type and has little relationship with the procedure.

Eight of 103 patients died. Six patients underwent 
Table 4 Analysis of influencing factors of improvement of postoperative muscle weakness in 54 patients with thymoma complicated with myasthenia gravis

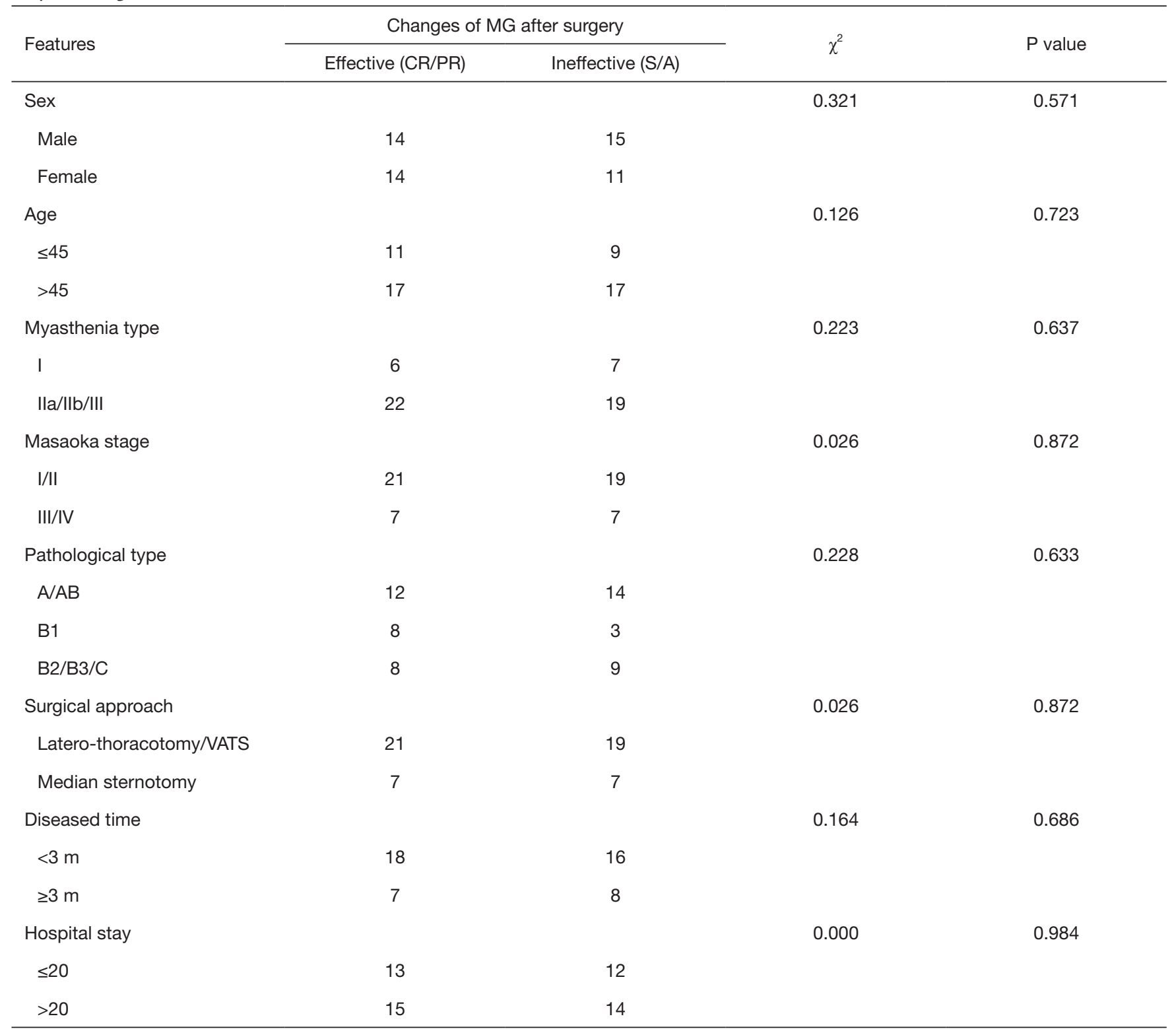

VATS, video-assisted thoracic surgery; MG, myasthenia gravis; S, stability; PR, partial remission; CR, complete remission; A, aggravation.

unilateral sacral nerve resection, among whom 4 patients developed respiratory dysfunction, and 3 died. Two patients died of MG after surgery, 1 patient died of tumor recurrence and metastasis, 1 patient died of heart disease, and the cause of death was unknown in the remaining patient. The remaining patients survived CT scans during the follow-up revealed tumor recurrence in 5 patients, among whom 2 patients underwent reoperation and 3 continued to receive radiotherapy. Since the other patients failed to receive regular CT scans during the follow-up, it was not possible to identify asymptomatic patients with local recurrence and thus the tumor-free survival could not be calculated. The overall accumulative survival is shown in Figure 1, and both 5- and 10-year survival rates were 91\% in the entire cohort. The 5 -year survival rate was slightly lower than that reported in literature, while the 10-year survival rate was comparable (28). Therefore, VATS do not increase surgical risk and will not negatively affect the 
Table 5 Clinical data of 41 patients with stage III/IV thymoma

\begin{tabular}{|c|c|c|}
\hline Features & \multicolumn{2}{|c|}{ Surgical approach } \\
\hline \multicolumn{3}{|l|}{ Sex } \\
\hline Male & $10(6 / 4)$ & 11 \\
\hline Female & $4(4 / 0)$ & 6 \\
\hline \multicolumn{3}{|c|}{ Myasthenia type } \\
\hline I & $2(2 / 0)$ & 1 \\
\hline $\mathrm{Ila}$ & $4(2 / 2)$ & 3 \\
\hline $\mathrm{Ilb}$ & $1(1 / 0)$ & 3 \\
\hline III & $11(7 / 4)$ & 14 \\
\hline IV & $3(3 / 0)$ & 3 \\
\hline \multicolumn{3}{|c|}{ Pathological type } \\
\hline$A$ & $3(2 / 1)$ & 1 \\
\hline$A B$ & $1(1 / 0)$ & 2 \\
\hline B1 & $3(2 / 1)$ & 0 \\
\hline B2 & $2(2 / 0)$ & 2 \\
\hline B3 & $3(2 / 1)$ & 2 \\
\hline \multicolumn{3}{|c|}{ Changes of MG after surgery } \\
\hline $\mathrm{CR}$ & $0(0 / 0)$ & 2 \\
\hline A & $3(2 / 1)$ & 3 \\
\hline Drainage time & $3.71 \pm 1.267(4.30 \pm 0.939 / 2.25 \pm 0.5)$ & $5.53 \pm 1.281$ \\
\hline Hospital stay & $20.50 \pm 9.549(23.60 \pm 8.984 / 12.75 \pm 6.397)$ & $27.29 \pm 9.531$ \\
\hline Tumor size* & $8.61 \pm 3.318(9.35 \pm 3.575 / 6.75 \pm 1.708)$ & $8.71 \pm 3.047$ \\
\hline
\end{tabular}

*, the longest diameter of the tumor. VATS, video-assisted thoracic surgery; MG, myasthenia gravis; S, stability; PR, partial remission; CR, complete remission; $A$, aggravation.

prognosis of patients.

According to our experience, the extent of thymectomy with resection of the anterior mediastinal fat tissue is generally as follows: left and right sides: to the pericardial sacral nerve level; lower border: to the adipose tissue at the palpebral angle; and the upper border: to the inferior thyroid plexus. The surgery within this extent can be easily completed via the median sternotomy incision. In patients undergoing conventional lateral thoracotomy, no median sternotomy is required during the dissection of anterior mediastinal fat tissue in the middle and lower sides of the contralateral side because excessive squeezing of the heart 


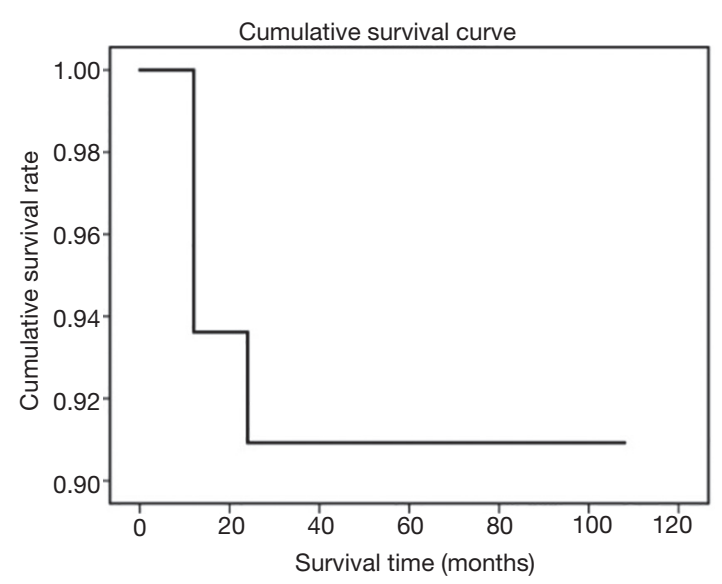

Figure 1 Survival curve of 103 patients.

is forbidden during the resection. In contrast, for patients undergoing VATS via a right-sized approach, the use of instruments and the enlarged visual field often enable the thorough dissection of anterior mediastinal fat tissue at the left side via the anterior cardiac space. Similarly, it has been proposed that unilateral or bilateral minimally invasive surgery can achieve the same extent of resection as median sternotomy $(15,29)$.

According to the results of our current study and our clinical experience, VATS is safe for thymomas that have not invaded the structures and organs other than the mediastinal pleura, have a longitudinal length of no more than $7 \mathrm{~cm}$, and have not severely compressed the innominate vein and can achieve the goal of radical cure. Therefore: (I) VATS surgery is similar to conventional surgeries in improving MG in thymoma patients with MG. (II) VATS resection can still be considered for thymoma that only invades the mediastinal pleura. (III) For thymomas that have intact capsules and have not invaded mediastinal pleura, an MT surgery shall be performed to ensure safety if their anteroposterior diameters are large and the masses have produced severe compression of the innominate vein, even if the tumors are still in the Masaoka stage II. For thymomas with large left-to-right diameters and with most parts of the tumors located in the left thoracic cavity, a leftsided approach (either VATS or an open approach) may be performed in the absence of MG; if MG complicates the condition, an MT approach or a bilateral VATS may be considered. In patients with unilateral pericardial phrenic nerve and/or local pericardial involvement, right-sided VATS thymectomy may be considered for thymomas located at the right side and bilateral VATS surgery can be performed for tumors located at the left side. In summary, VATS is feasible for the treatment of thymoma complicated by MG. VATS can be performed in patients with Masaoka stage I, II and (a certain portion of) III thymoma; for some patients with Masaoka stage II thymoma, especially those with compression of the innominate vein, the use of VATS should be cautious.

\section{Acknowledgments}

None.

\section{Footnote}

Conflicts of Interest: The authors have no conflicts of interest to declare.

Ethical Statement: The authors are accountable for all aspects of the work in ensuring that questions related to the accuracy or integrity of any part of the work are appropriately investigated and resolved. This paper is a retrospective study, no artificial intervention was applied in the treatment of the whole group of cases, and no different treatment methods were deliberately adopted for patients to obtain a certain group of data. All patients were treated according to the actual clinical needs. Therefore, we state that this study does not involve the content requiring ethical review.

\section{References}

1. Engels EA, Pfeiffer RM. Malignant thymoma in the United States: demographic patterns in incidence and associations with subsequent malignancies. Int J Cancer 2003;105:546-51.

2. Maniscalco P, Tamburini N, Quarantotto F, et al. Longterm outcome for early stage thymoma: comparison between thoracoscopic and open approaches. Thorac Cardiovasc Surg 2015;63:201-5.

3. Blalock A, Mason MF, Morgan HJ, et al. Myasthenia gravis and tumors of the thymic region: report of a case in which the tumor was removed. Ann Surg 1939;110:544-61.

4. Keynes G. The surgery of the thymus gland. Br J Surg 1946;33:201-14.

5. Sonett JR, Jaretzki A 3rd. Thymectomy for nonthymomatous myasthenia gravis: a critical analysis. Ann N Y Acad Sci 2008;1132:315-28.

6. Peel JK, Prisma E, Nq TL, et al. Ectopic thymoma 
managed by neck dissection \& video-assisted thoracoscopic thymectomy. J Thorac Dis 2017;9:E1050-3.

7. Xue L, Pang X, Zhang Y, et al. Extend thymectomy by a cervical incision additional to bilateral VATS approach. J Vis Surg 2017;3:83.

8. Migliore $M$, Criscione A, Nardini $M$, et al. Single incision extended video assisted transcervical thymectomy. J Vis Surg 2017;3:154.

9. Infante $M$, Benato C, Giovannetti R, et al. VATS thymectomy for early stage thymoma and myastenia gravis: combined right-sided uniportal and left-sided three-portal approach. J Vis Surg 2017;3:144.

10. Marulli G, Comacchio GM, Rea F. Robotic thymectomy. J Vis Surg 2017;3:68.

11. Rea F, Schiavon M, Marulli G. Robotic thymectomy for myasthenia gravis. Ann Cardiothorac Surg 2015;4:558-60.

12. Sugarbaker DJ. Thoracoscopy in the management of anterior mediastinal masses. Ann Thorac Surg 1993;56:653-6.

13. Takeo S, Sakada T, Yano T. Video-assisted extended thymectomy in patients with thymoma by lifting the sternum. Ann Thorac Surg 2001;71:1721-3.

14. Cheng YJ, Kao EL, Chou SH. Videothoracoscopic resection of stage II thymoma: prospective comparison of the results between thoracoscopy and open methods. Chest 2005;128:3010-12.

15. Pennathur A, Qureshi I, Schuchert MJ, et al. Comparison of surgical techniques for early stage thymoma: feasibility of minimally invasive thymectomy and comparison with open resection. J Thorac Cardiovasc Surg 2011;141:694-701.

16. Falkson CB, Bezjak A, Darling G, et al. The management of thymoma: a systematic review and practice guideline. J Thorac Oncol 2009;4:911-9.

17. Detterbeck FC, Parsons AM. Thymic tumours. Ann Thorac Surg 2004;77:1860-9.

18. Agasthian T, Lin SJ. Clinical outcome of video-assisted thymectomy for myasthenia gravis and thymoma. Asian Cardiovasc Thorac Ann 2010;18:234-9.

Cite this article as: Wang GW, Tao T, Li CK, Li QC, Duan GX, Sang HW, Dong HJ, Wang ZY. Comparison between thoracoscopic and open approaches in thymoma resection. J Thorac Dis 2019;11(10):4159-4168. doi: 10.21037/ jtd.2019.09.85
19. Odaka M, Akiba T, Mori S, et al. Oncological outcomes of thoracoscopic thymectomy for the treatment of stages I-III thymomas. Interact Cardiovasc Thorac Surg 2013;17:285-90.

20. Venuta F, Rendina EA, Anile M, et al. Thymoma and thymic carcinoma. Gen Thorac Cardiovasc Surg 2012;60:1-12.

21. Toker A, Sonett J, Zielinski M, et al. Standard terms, definitions, and policiesfor minimally invasive resection of thymoma. J Thorac Oncol 2011;6:S1739-42.

22. Narm KS, Lee CY, Do YW, et al. Limited thymectomy as a potential alternative treatment option for early-stage thymoma: A multi-institutional propensity-matched study. Lung Cancer 2016;101:22-7.

23. Nakagawa K, Yokoi K, Nakajima J, et al. Is thymomectomy alone appropriate for stage I (T1N0M0) thymoma? Results of a propensity-score analysis. Ann Thorac Surg 2016;101:520-6.

24. Onuki T, Ishikawa S, Iguchi K, et al. Limited thymectomy for stage I or II thymomas. Lung Cancer 2010;68:460-5.

25. Tseng YC, Hsieh CC, Huang HY, et al. Is thymectomy necessary in nonmyasthenic patients with early thymoma? J Thorac Oncol 2013;8:952-8.

26. Bae MK, Lee SK, Kim HY, et al. Recurrence after thymoma resection according to the extent of the resection. J Cardiothorac Surg 2014;9:51.

27. Nakagawa K, Asamura H, Sakurai H, et al. Does the mode of surgical resection affect the prognosis/recurrence in patients with thymoma? J Surg Oncol 2014,109:179-83.

28. Safieddine N, Liu G, Cuningham K, et al. Prognostic factors for cure, recurrence and long-term survival after surgical resection of thymoma. J Thorac Oncol 2014;9:1018-22.

29. Lee CY, Kim DJ, Lee JG, et al. Bilateral video-assisted thoracoscopic thymectomy has a surgical extent similar to that of transsternal extended thymectomy with more favorable early surgical outcomes for myasthenia gravis patients. Surg Endosc 2011;25:849-54. 